\title{
ON GEOMETRY OF SOLUTIONS TO APPROXIMATE EQUATIONS AND THEIR SYMMETRIES
}

\author{
V.V. GORBATSEVICH
}

\begin{abstract}
The paper is devoted to developing a geometric approach to the theory of approximate equations (including ODEs and PDEs) and their symmetries. We introduce dual Lie algebras, manifolds over dual numbers and dual Lie group. We describe some constructions applied for these objects. On the basis of these constructions, we show how one can formulate basic concepts and methods in the theory of approximate equations and their symmetries. The proofs of many general results here can be obtained almost immediately from classical ones, unlike the methods used for studying the approximate equations.
\end{abstract}

Keywords: approximate equation, approximate Lie algebra, dual numbers, dual Lie algebra, manifold over dual numbers.

Mathematics Subject Classification: 53C15, 34C30, 37J15, 51H30

\section{INTRODUCTION}

In the paper we consider notions arising in studying approximate symmetries of various functional and differential objects depending on a small parameter. This direction is initiated by paper [1] and then it was continued in many other works. The main aim of the present paper is not to study new examples or to calculate the symmetries for new equations, but to construct a geometric approach for studying these equations and symmetries and to develop the corresponding methodology. Here the principle of the authors was that advocated by A. Grothendieck: "In order to solve a problem, it should be embedded in such mathematical environment, in which its solution becomes obvious". The paper is devoted to describing exactly such environment, in which approximate equations and their symmetries are formulated and solved in a most natural way. In particular, we shall prove a rather general statement, a correspondence principle, which can be formulated as follows.

Let $F(x, u, \epsilon)=0$ be some equation (probably, an ordinary or partial differential equation), where $u$ is a smooth scalar or vector function of a scalar or vector independent variable $x$, and $\epsilon$ is a parameter, which is usually regarded as close to zero. We consider the linearization $F(x, u) \approx f_{0}(x, u)+\epsilon f_{1}(x, u)$ of the left hand side of this equations and continue it to the domain of dual values of the arguments, that is, to the dual numbers $a+\epsilon b$, where $a, b \in \mathbb{R}$, $\epsilon^{2}=0$. We obtain the equation $\tilde{F}(\tilde{x}, \tilde{u})=0$. Let $\tilde{u}(\tilde{x})$ be its exact solution. Then restricting it on the real values $\tilde{x}=x$, we obtain an approximate solution to the original equation up to $o(\epsilon)$.

The most general formulation of the main methodological result is very elementary: two functions $\Phi(*, \epsilon)$ and $\Psi(*, \epsilon)$ are equal up to $o(\epsilon)$ if and only if the associated functions $\Phi(\tilde{*})$

V.V. Gorbatsevich, On GeOMEtry of SOlutions to APproximate EQuations And their symmeTRIES.

(C) Gorbatsevich V.V. 2017.

Submitted July 14, 2016. 
and $\Psi(\tilde{*})$ of the dual arguments are equal. Applying this to the solutions of a differential equation, we obtain the above formulated correspondence principle.

The notions used in these formulations will be described in details in what follows. The general correspondence principle is applicable, in particular, to the solutions of differential depending on a parameter as well as to calculating the symmetries for these equations.

In Section 1 we formulate the main notions related to the methods of solving approximate equations and calculating their symmetries. We consider versions of defining approximate Lie algebras, one of the main notions in the further exposition.

In Section 2 we consider the notions of a symmetry and an approximate symmetry for equations.

In Section 3 we give the definition of an approximate Lie algebra, which is related with the notion of Lie algebra over the algebra of dual numbers $D_{2}$.

In Section 4 we introduce the notion of $D_{2}$-manifold being the main one for the present paper and providing a geometric base for studying approximate equations and their symmetries. We introduce also usual notions accompanying a manifold: the tangent vectors and fields, etc.

In Section 5 we prove some results of general theory of differential equations with a dual argument.

Section 6 is devoted to a brief discussion of Lie theory in $D_{2}$-situation.

And finally, in Section 7 we consider application of the said above to the issue on the methods of solving and analysing approximate solutions. We give a geometric interpretation of one widely used method for solving an approximate solution and point out a generalization of this method. As a demonstration, we consider a simple example of solving the Cauchy problem for an approximate second order ordinary differential equation showing the usefulness of passing to $D_{2}$-domain.

\section{Motivation AND INITIAL NOTION}

As it is known, Sophus Lie started his studies on the theory of continuous transformations groups trying to extend the methods of Galois theory on the differential equations. At that, he needed to introduce the notion of the symmetry group of a differential equation and some other functional and differential objects. Then, in a modern language, the notion of Lie transformations groups was introduced as well as of the corresponding Lie algebra of vector fields. And only after that, the general notions of Lie group (not necessarily transformation group) and of abstract Lie algebra were introduced in the mathematics.

The initial notion of symmetries group (in fact, this was an one-parametric transformation group) preserving a function or a differential equation was studied rather in details and there was considered many times its various applications in the theory of differential equations, in geometry, in theoretical physics, etc. But in practical applications certain difficulties arose. The most part of differential equations in physics, mechanics and other fields of applying the mathematics are approximate and involve some parameters, usually small ones. The vanishing of one of the parameters corresponds to certain "classical" situation. But real physical and other objects are described more precisely for some non-zero and small values of these parameters. The typical examples of a parameter of such kind is the Planck constant $\hbar$ or the quantity $1 / c$, where $c$ is the light speed. As $c \rightarrow \infty$ we have $1 / c \rightarrow 0$ and the passage from the relativistic theory to the classical physics. As $\hbar \rightarrow 0$, the quantum mechanics becomes the classical one. Such small parameters gave rise to the notion of quantization and in the most general situation, this notion means some deformation depending on a parameter. Under such "quantizations", the symmetries groups change and at that, some of the symmetries are "destroyed". This showed the need of studying the symmetries, which do not disappear for non-zero values of a parameter but "deform" somehow. This is how the method of studying "approximate" 
symmetry appeared. There are several approaches to the notion of approximate symmetry of a differential equation. In this work we base on that introduced in [1] and it was introduced in details by a series of authors (see, for instance, surveys [2], 3]). However, our approach differs a little from that employed by these authors and our approach is more geometric and is based on the notion of a manifold over the algebra of dual numbers.

In what follows we employ the standard notation $f \approx g$ for the equivalence of two functions in the variable $\epsilon$ : the writing $f(x, \epsilon) \approx g(x, \epsilon)$ stands for the condition $f(x, \epsilon)-g(x, \epsilon)=o(\epsilon)$ as $\epsilon \rightarrow 0$. We can also consider $o\left(\epsilon^{p}\right)$ for an arbitrary natural $p$, but here we usually do not use it pointing out sometimes possible generalizations.

We begin with preliminary ideas and definitions introduced and studied first in [1]. Assume that we have the equation $F(x, \epsilon)=0$, where $F$ is some smooth or even analytic function depending on a variable $x$, which can be a vector one, $x=\left(x_{1}, x_{2} \ldots\right)$, and on a small parameter $\epsilon$. The value $\epsilon=0$ corresponds to the equation $f_{0}(x)=0$ (where $f_{0}(x)=F(x, 0)$ ), which will be referred to as exact, while the initial equation will be referred to as approximate. In the same way we can consider systems of equations (as $F$ is a vector-value mapping) depending on a scalar parameter, as well as differential equations, for instance, $F\left(x, u, u^{\prime}, \ldots, \epsilon\right)=0$ or $F\left(x, y, u, u_{x}, u_{y}, \ldots, \epsilon\right)=0$.

Now we consider the notion of an one-parametric approximate transformations group and its infinitesimal analogue, an approximate vector field or, equivalently, an approximate differential operator corresponding to this group. All considerations will be local and this is why we can suppose that all objects are defined in some neighbourhood of the origin in the Euclidean space $\mathbb{R}^{n}$. At that, we shall follow the classical definitions in the theory of the transformations groups.

An one-parametric (local) transformations group is a mapping $x^{\prime}=\phi(x, a)$ depending on $a \in \mathbb{R}$ (the parameter $a$ can also ranges in a symmetric interval in a local situation) and for each $a, b \in \mathbb{R}$ satisfying two identities

$$
\begin{aligned}
& \phi(\phi(x, a), b)=\phi(x, a+b), \\
& \phi(x, 0)=x .
\end{aligned}
$$

We note that the inverse transformation always exists and it corresponds to the value $-a$ of the parameter. The vector field $\mathcal{X}$ associated with this one-parametric transformations groups (sometimes it is called the infinitesimal transformation or generator) is defined as follows: it is of the form $\mathcal{X}=X(x) \frac{d}{d x}$, where

$$
X(x)=\frac{d}{d a}(\phi(x, a))_{a=0}
$$

The invariance of a function $F(x)$ w.r.t. an one-parametric transformations group is introduced in a natural way:

$$
\phi(F(x), a)=F(x)
$$

for each admissible $a \in \mathbb{R}$.

As it is known from the times of S. Lie, this condition is equivalent to the vanishing of the action of the corresponding differential operator to this function, that is, to the condition $\mathcal{X} \cdot F=0$.

Now we consider the situation, when we deal with a small parameter $\epsilon$. Here the same constructions are reproduced but an additional variable aries, the parameter $\epsilon$, and all identities are replaced by "approximate identities". In particular, an approximate one-parametric (local) transformations group is a mapping such that $x^{\prime} \approx \phi(x, a)$, where $a \in \mathbb{R}$ (or $a$ ranges in some 
interval in the local situation), and for each $a, b \in \mathbb{R}$ the identities

$$
\begin{aligned}
& \phi(\phi(x, a), b) \approx \phi(x, a+b), \\
& \phi(x, 0) \approx x .
\end{aligned}
$$

In these relations the presence of the small parameter $\epsilon$ is assumed.

The approximate invariance condition for a function $F($ as $\phi(F(x), a) \approx F(x)$ for each $a \in \mathbb{R})$ casts into the form $\mathcal{X} \cdot F \approx 0$. Here $\mathcal{X}=\mathcal{X}(x, \epsilon)$ is a vector field smooth or analytic subject to the considered situation depending on the parameter $\epsilon$. At that, $\mathcal{X}(x, \epsilon)$ can be considered as the one-parametric family (depending on the parameter $\epsilon$ ) of "exact" vector field.

For differential equations the notions of symmetry and approximate symmetry are introduced in the natural way and at that, the natural continuation of the coordinates transformation on the derivatives is employed.

When we pass from one-parametric transformations groups to the complete symmetries group (being Lie groups, probably, infinitely dimensional), the corresponding infinitesimal transformations form Lie algebras of vector fields. Under the presence of the parameter $\epsilon$ the arising families of vector field corresponding to the approximate symmetries do not form, generally speaking, Lie algebras since the axioms of Lie algebra (in particular, Jacobi condition) hold only approximately. The associated infinitesimal objects are naturally called approximate Lie algebras and we shall dwell on them below.

The considering of functions and vector fields up to $o(\epsilon)$ is equivalent to the linearization w.r.t. the parameter $\epsilon$. In particular, the function $F(x, \epsilon)$ is replaced by the function $f_{0}(x)+\epsilon f_{1}(x)$, where $f_{0}, f_{1}$ are some smooth or analytic functions. For an approximate vector field $\mathcal{X}(x, \epsilon)$ we have

$$
\mathcal{X}(x, \epsilon) \approx \mathcal{X}_{0}(x)+\epsilon \mathcal{X}_{1}(x),
$$

where $\mathcal{X}_{0}, \mathcal{X}_{1}$ are usual vector fields. The commutation of approximate vector fields goes as follows:

$$
[\mathcal{X}, \mathcal{Y}]=\left[\mathcal{X}_{0}+\epsilon \mathcal{X}_{1}, \mathcal{Y}_{0}+\epsilon \mathcal{Y}_{1}\right]=\left[\mathcal{X}_{0}, \mathcal{Y}_{0}\right]+\epsilon\left(\left[\mathcal{X}_{0}, \mathcal{Y}_{1}\right]+\left[\mathcal{X}_{1}, \mathcal{Y}_{0}\right]\right)
$$

At that, the bilinearity and skew-symmetricity are preserved and the Jacobi identity holds. Hence, we obtain some Lie algebra. But if we consider the usual commutator of linearized vector fields, it will not be linear in $\epsilon$. This is why the above mentioned commutation is not the commutation of vector fields, it is made in a slightly different way. We arrive at a new notion, in which all Lie algebras depending on the parameter $\epsilon$ are united into a single object. Following [4], this object $\mathcal{X}(\epsilon)$ is called approximate Lie algebra and now we consider several different approaches to considering such objects.

The first approach is as follows. Let $\Phi$ be some Lie algebra of vector fields (of their germs) in some neighbourhood of a point $x \in \mathbb{R}^{n}$. We consider the set of the vector fields of form $X+\epsilon Y$, where $X, Y \in \Phi$, and $\epsilon$ is some parameter. We denote this set by $\Phi(\epsilon)$ and we represent it as $\Phi+\epsilon \Phi$. Generally speaking, this set of (the germs of) vector fields do not form a Lie algebra since the commutation can give rise to vector fields outside $\Phi(\epsilon)$ since there can arise a term with $\epsilon^{2}$. But we define the commutation on $\Phi(\epsilon)$ in a different way (as it was done above for approximate vector fields), as one can easily confirm, we obtain a Lie algebra but it will not the Lie algebra of vector fields. In the above cited papers such Lie algebras are called approximate.

Apart of the approximate Lie algebras constructed in this way, it is useful to consider some of their subalgebras; not all of them but only those passing the multiplication by $\epsilon$. Exactly such Lie subalgebras correspond to Lie groups of approximate symmetries of differential equations and the studying of these equation initiated the introducing of notion of approximate Lie algebra. 
We also observe that considering of the expressions of similar form $\bar{a}+\omega \bar{a}^{0}$, where $\bar{a}, \bar{a}^{0}$ are three-dimensional vectors and $\omega$ is a symbol satisfying $\omega^{2}=0$ employed in the mechanics as the object of screw theory (see, for instance, [5]).

Now we consider another construction. Let $\Phi$ be an arbitrary real Lie algebra. We consider the two-dimensional algebra $D_{2}$ (the algebra of dual numbers of the form $a+\epsilon b$ called sometimes Studi algebra and its elements are sometimes called Studi numbers) over $\mathbb{R}$ with generators 1 and $\epsilon$ and $\epsilon^{2}=0$. We note that the algebra $D_{2}$ (commutative, associative and having the unit) has an exact matrix representation, whose matrices are of the form $\left(\begin{array}{cc}a & b \\ 0 & a\end{array}\right)$, where $a, b \in \mathbb{R}$. It is interesting to mention that in distinction to the field $\mathbb{R}$, whose group of automorphisms is trivial, and to the field of complex numbers $\mathbb{C}$, whose group of automorphisms is isomorphic to $\mathbb{Z}_{2}$ (with the only nontrivial automorphism, which is the complex conjugation), the algebra $D_{2}$ has an one-dimensional group of automorphisms. This group is formed by the transformations of the form $a+\epsilon b \rightarrow a+\epsilon \alpha b$ with arbitrary nonzero $\alpha \in \mathbb{R}$.

We let $\tilde{\Phi}=\Phi \otimes D_{2}$, where the right hand side is the tensor product of the algebras $\Phi$ and $D_{2}$. We can write the elements $\tilde{\Phi}$ as $a+\epsilon b$, where $a, b \in \Phi$. The commutation in the algebra $\tilde{\Phi}$ is introduced in the natural way (commutation in the first tensor factor and the multiplication in the second tensor factor), at that we obtain the structure of the Lie algebra. In fact, $\tilde{\Phi}$ can be considered as the semi-direct sum $\Phi+{ }_{a d} \Phi$ of a subalgebra isomorphic to $\Phi$ and an Abel ideal corresponding to adjoint action $a d$ of the Lie algebra $\Phi$ on itself as on a vector space. It is clear that if $\Phi$ is the Lie algebra of vector field, by such construction we obtain the approximate Lie algebra in the previous construction.

This construction can be generalized. For instance, consider the algebra $\left.D_{p}=\mathbb{R}[\epsilon] /<\epsilon^{p}\right\rangle$, where $\mathbb{R}[\epsilon]$ is the algebra of polynomials in $\epsilon$, and $\left\langle\epsilon^{p}\right\rangle$ is the ideal generated by the element $\epsilon^{p}$. We obtain an associative and commutative algebra with a unity of dimension $p$ over $\mathbb{R}$, sometimes it is called the algebra of plural numbers. As $p=2$, this algebra has already been introduced above. Now we consider the tensor product $\Phi \otimes D_{p}$ with the natural commutation; the obtained Lie algebra can be considered as an approximate Lie algebra while employing approximations of order $p$ by means of $o\left(\epsilon^{p}\right)$. It is represented as the semi-direct sum of a subalgebra isomorphic to $\Phi$ and an nilpotent ideal of the form $\epsilon \Phi+\epsilon^{2} \cdot \Phi \cdots+\epsilon^{p-1} \cdot \Phi$.

\section{ON SYMMETRIES OF EQUATIONS}

We proceed to a more detailed studying of approximate symmetries of equations. Our main aim is to develop of geometric approach and the methods for studying approximate groups of symmetries of ordinary and partial differential equations. We begin with approximate solving the equations. We shall make our considerations at the simplest example: we consider a first order ordinary differential equation and the Cauchy problem on some segment $(a, b)$ :

$$
\begin{aligned}
& u^{\prime}=F(x, u, \epsilon), \\
& \left.u\right|_{x=x_{0}}=u^{0} .
\end{aligned}
$$

Here $u(x)$ is an unknown function. As $\epsilon=0$, we refer to the equation as exact.

We consider the group Diff (infinite-dimensional Lie group) of local diffeomorphisms of the range of the variable $x$ (a neighbourhood of the point $x_{0}$ ). It acts on sought functions $u$ and on the entire differential equations. The stationary subgroups of this action for each $\epsilon$ are exactly the (local) Lie groups of the symmetries of the considered differential equations for given $\epsilon$. For simplicity we assume that these groups of symmetries are finite-dimensional that often turns out for important and interesting differential equations. Then, as it is known, for small $\epsilon$ the (finite) dimension of a stationary subgroup does not exceed that of the stationary subgroup as $\epsilon=0$ (that is, under the deformation of a "point" the dimension of its stationary subgroup does not increase). This phenomenon is studied in great details while treating the symmetries of 
differential equations. If the dimension is constant for small $\epsilon$, one says about stable symmetries and a possible continuation of the symmetry of the equation corresponding to $\epsilon=0$ (that is, of the exact equation). If the dimension of the group of symmetries decreases for small $\epsilon \neq 0$, one says that some symmetries do not admit the continuation and this is why even for the exact equation there is no need to consider them, that is, they depend unstable on the parameter. It is useful to note that the stability property for the group of symmetries for different, even close equations can show in a different way. We stress that above we discussed the groups of symmetries treated in the usual exact meaning. In fact, one considers also approximate symmetries, for which one can not applied straightforwardly the above mentioned arguments on the dimension of stationary subgroups. So, the above discussions on the character of varying of the dimension of symmetries group should be regarded mostly as heuristic arguments.

For studying differential equations depending on a parameter and for finding approximate Lie groups and their symmetries, there were proposed two methods having rather precise geographic locations.

One of these methods developed originally in Ufa mathematical school is based on the linearization of equations and differential operators of symmetry (by means of considering them up to $o(\epsilon)$; sometimes higher order approximations are used). Here there arise principally new mathematical objects called approximate transformations groups with associated approximate Lie algebras. By reproducing classical proofs in the "exact" case there were proved an analogue of exact Cauchy theorem on existence of approximate solution for approximate Cauchy problem and other analogues of classical results. Approximate symmetries of the obtained approximate equations are studied intensively.

The second method was created in Kiev mathematical school and it is more classical. Here one considers the expansion of the unknown functions w.r.t. the parameter. For the obtained approximate equations the exact classical symmetries are considered.

The application of these two methods to particular equations often give different results. There are several works (for instance, [6]), where it is found out which of these methods give more precise results at the example of a series of differential equations in various branches of physics and mechanics for which one can calculate exact symmetries groups for arbitrary values of the parameters. In [6] the second method is preferred but however, this method became less widespread among the specialists. At that it should be noticed that the symmetries groups for the differential equations is a rather gentle object and is very sensible to the nature of the studied equation and the choice of the small parameter. This is why a "statistical analysis" seems to be not very appropriate while considering the efficiency of these two methods.

As it was mentioned above, in the present paper we study exactly the first method and the related new mathematical objects; the second method is much poorer in the latter sense.

\section{Approximate AND DUAL Lie ALGEBras}

It was mentioned above that we can introduce the notion of the approximate Lie algebra of vector fields. Here the usual conditions defining Lie algebras are replaced by their approximate analogues. But it is more natural to pass to more general constructions and this is what we do.

First as approximate Lie algebras we call Lie algebras of the form $L \otimes D_{2}$, which are the tensor products of usual Lie algebras $L$ and the two-dimensional algebra $D_{2}=<1, \epsilon>$ (the algebra of dual numbers). We note that such Lie algebras can be also considered as $D_{2}$-moduli. Moreover, approximate Lie algebras of the symmetries of approximate differential equations also have the structure of $D_{2}$-moduli. This is implied by the following simple fact: if a vector field $X$ is an infinitesimal symmetry of a differential equation, then the same is true for the vector field $\epsilon X$. Hence, the Lie algebra of infinitesimal symmetries is invariant w.r.t. the multiplication by the elements of the algebra $D_{2}$. 
Apart of the Lie algebra of the form $L \otimes D_{2}$, one can and one should consider their subalgebras. But if we are interested in Lie algebras of approximate symmetries, it is natural to consider only the subalgebras being $D_{2}$-moduli. And exactly these subalgebras are to be called approximate Lie algebras in the sense arising in studying approximate differential equations and their symmetries. So, we arrive at the need to consider the class of all Lie algebras defined over $D_{2}$. Exactly them we call approximate Lie algebras. Thus, here is the final definition.

Definition 1. An approximate Lie algebra is the Lie algebra over the algebra $D_{2}$ of dual numbers. In other words, this is a Lie algebra, which in addition possesses the structure of $D_{2}$-module compatible with the commutation.

At that, the structure of $D_{2}$-module is defined by a linear operator corresponding to the multiplication by $\epsilon$. We denote this operator by $\mathcal{E}$. It is natural to study the corresponding (invariant w.r.t. $\mathcal{E}$ ) Lie subalgebras, ideal, homorphisms, etc. We note that a $D_{2}$-module of a Lie algebra can be trivial (as the operator $\mathcal{E}$ is zero). Thus, the usual Lie algebras, for which $\mathcal{E}=0$, can be considered as Lie $D_{2}$-algebras. However, in what follows we mostly assume that $\mathcal{E} \neq 0$. Moreover, sometimes it is useful to consider only "non-degenerate" $D_{2}$-structures, when the rank of the operator $\mathcal{E}$ is equal to the half of the dimension of the Lie algebra, which is of course assumed to be even-dimensional. The Lie algebras of the form $L \otimes D_{2}$ are exactly of such kind.

As a basis of Lie $D_{2}$-algebra, one treats the minimal set of the vectors generating this Lie algebra as a $D_{2}$-module. In other words, this is a minimal set of vectors which together with the multiplications by $\epsilon$ generate Lie algebra as a vector field over $\mathbb{R}$. The bases of such kind were introduced in [4] and called there as essential. We note that the notion of the essential basis makes sense only for Lie algebras over $D_{2}$. The number of the elements in this basis is a little bit non-standard analogue of the dimension of the considered Lie algebra over $D_{2}$. For instance, Lie subalgebra in $L \otimes D_{2}$ generated by the element of the form $\epsilon X$ is one-dimensional over $\mathbb{R}$, while that generated by the element $X \in L$ is two-dimensional.

As examples of $D_{2}$-algebras, we can take Lie algebras of the form $L \otimes D_{2}$ for arbitrary real Lie algebras. In particular, if Lie algebra $L$ is linear, in the tensor product of this matrix by $D_{2}$, the matrix elements of this matrix are taken lying in $D_{2}$ (with the relations corresponding to the Lie algebra $L)$. For instance, if $L=\operatorname{sl}(n, \mathbb{R})$, we obtain the Lie algebra $\operatorname{sl}\left(n, D_{2}\right)$ consisting of the matrices of order $n$ with the entries in $D_{2}$ and the trace of these matrices is zero. We note that is the Lie algebra $L$ is semi-simple, then $L \otimes D_{2}$ is never semi-simple (all Lie algebras of the form $L \otimes D_{2}$ have non-zero radical).

\section{Smooth manifolds with $D_{2}$-Structure}

Having introduced Lie algebras over $D_{2}$, it is natural not to stop and to consider smooth manifolds with additional $D_{2}$-structure. This will allow us to consider approximate differential equations and many related notions (in particular, groups of approximate symmetries) in a more natural way employing geometric methods.

We note that while studying approximate equations we could not leave beyond usual manifolds and this was done till now. Employing the relation $\approx$, we can introduce the notion of the approximate Lie algebra of vector fields; this is a set of vector fields, which is closed w.r.t. the commutation up to $o(\epsilon)$ and satisfies usual axioms of the Lie algebra up to $o(\epsilon)$. But such approach seems to be artificial and we shall not develop it.

We proceed to describing the notion of $D_{2}$-manifolds and related notions (tangent spaces, mappings, Lie groups, etc). We note that the corresponding geometric constructions were developed in details in Kazan mathematical school [7]. 
We consider the notion of a manifold over the algebra of dual numbers (the constructions over the algebras of plural numbers are similar but we do not dwell on them). The manifolds are defined by the standard scheme, the charts diffeomorphic to domains in $D_{2}^{n}$ are glued by means of transition maps preserving $D_{2}$-structure. Let us give a more detailed description.

Let us consider the space $D_{2}^{n}$. This is a free $D_{2}$-module over the algebra $D_{2}$ having $n$ generators. As a vector space over $\mathbb{R}$, it is isomorphic to $\mathbb{R}^{2 n}$. But in distinction to the space $\mathbb{R}^{2 n}$, its elements admit the multiplication by the elements $a+\epsilon b$ of the dual algebra $D_{2}$. This defines $D_{2}$-structure on it, for instance, be means of the operator $\mathcal{E}$.

A smooth mapping $f: U \rightarrow V$ of the domain $U \subset D_{2}^{n}$ into the domain $V \subset D_{2}^{m}$ is called smooth $D_{2}$-mapping (sometimes the terms "holomorphic" or "analytic" are employed similar to the complex case) if its differential preserves the natural $D_{2}$-structure on tangent spaces identified with $D_{2}^{n}$ and $D_{2}^{m}$, respectively. In other words, the operator of the multiplication by $\epsilon$ should commute with the differential.

We consider briefly the condition of $D_{2}$-smoothness or "holomorphy" for the function of one variable (in the general case, these are Scheffers conditions, who studied first smooth mappings over algebras and which analogues of Cauchy-Riemann conditions). The holomoprhy condition for the function $F(z)=f(x, y)+\epsilon g(x, y)$ of the dual variable $z=x+\epsilon y$ can be written as a condition for the differential, which is of the form $d F=(A+\epsilon B) \cdot d z$ (where $A=A(z), B=B(z) \in \mathbb{R}$ for a fixed value of the argument $z$ ). Opening brackets and equating the real and "imaginary" parts, we obtain:

$$
f_{x}^{\prime}=A, \quad g_{x}^{\prime}=B, \quad f_{y}^{\prime}=0, \quad g_{y}^{\prime}=A .
$$

This implies that $f(x, y)=\phi(x)$ is an arbitrary function of $x$ and $g(x, y)=y \cdot \phi_{x}+\psi(x)$, where $\psi(x)$ is one more arbitrary function of $x$. In this way we obtain the general form of $D_{2}$-smooth functions of one variable:

$$
F(x+\epsilon y)=\phi(x)+\epsilon\left(y \cdot \phi_{x}+\psi(x)\right) .
$$

We note that $y=0$, that is, if the independent variable is real, then the values of a $D_{2^{-}}$ smooth function are of the form $F(x)=\phi(x)+\epsilon \psi(x)$, that is, $F(x)$ is an arbitrary smooth vector function of $x$. This implies that a function of dual independent variable is uniquely determined by its values for real independent variable. If we consider $F(x+\epsilon y)$ as a vector function of two variables, its Jacobi matrix is of the form:

$$
\left(\begin{array}{cc}
\phi^{\prime}(x) & 0 \\
-y \phi^{\prime}(x)+\psi^{\prime}(x) & \phi^{\prime}(x)
\end{array}\right) .
$$

It is clear that this matrix commutes with the matrix $\left(\begin{array}{ll}0 & 0 \\ 1 & 0\end{array}\right)$ of the operator $\mathcal{E}$ on $\mathbb{R}^{2}$ that corresponds to the definition of a differential function of the dual independent variable.

Since as the components of a $D_{2}$-smooth function arbitrary smooth real functions can serve, it is clear that $D_{2}$-smooth functions not always can be expanded into power series, which can be introduced by means of the multiplicative norm on $D_{2}$ mentioned below. This is way the name "holomorphic" for $D_{2}$-functions seems to be not very apt since by analogy with the theory of function of complex variable it suggest the analiticity, which in $D_{2}$-situation can be absent.

In the same way one can obtain the general form of $D_{2}$-smooth function of several variables; for instance, one can read about this in [7].

We note that by means of the Taylor expansion we can construct very naturally the continuation of usual smooth functions into the dual domain of the values of the independent variable. For a function $u(x)$ such continuation will be denoted by $\tilde{u}(\tilde{x})$. We recall that the expressions of the form $\bar{a}+\omega \bar{a}^{0}$, where $\bar{a}, \bar{a}^{0}$ are three-dimensional vectors and $\omega$ is the symbol satisfying $\omega^{2}=0$ are used in the mechanics as the object of screw theory, see [5]. Exactly from the 
screw theory many formulas were borrowed in studying the functions of the dual independent variable.

We proceed to defining $D_{2}$-manifolds. A topological space $M$ is called smooth $D_{2}$-manifold if it is a second countable Hausdorff space and it has an atlas with charts in $D_{2}^{n}$, whose transition maps are $D_{2}$-smooth functions. But we can do it in another way: let $M$ be a usual evendimensional smooth manifold. It is called $D_{2}$-manifold (or a manifold with the $D_{2}$-structure) if for some atlas (set of charts) the corresponding transition maps are $D_{2}$-smooth. For $D_{2^{-}}$ manifolds, their tangent spaces have $D_{2}$-structure. The mappings in this category are smooth mappings preserving $D_{2}$-structure (in particular, the whose differentials are $D_{2}$-mappings). Employing the notion of the operator $\mathcal{E}$ corresponding to $D_{2}$-structure, the smoothness condition for the mapping $f: M \rightarrow N$ is written as $d f\left(\mathcal{E}_{M}\right)=\mathcal{E}_{N}(d f)$.

We note that for an arbitrary smooth $D_{2}$-manifold $M$, the space $T(M)$ of its tangent bundle (on tangent vectors see below) has a natural $D_{2}$-structure (for more details on $D_{p}$-structures on tangent spaces of higher orders see, for instance, [7]). In particular, a $D_{2}$-manifold can be considered as a smooth manifold, for which the tangent bundle over an arbitrary Euclidean space can serve as a model.

We also note that to define a $D_{2}$-topology we can employ the usual metric in the Euclidean space. However, in what follows we shall need the existence of a multiplicative norm in $D_{2}$. As it is known, the existence of the multiplicative norm is proven for arbitrary Banach algebras and this is why it holds for the two-dimensional algebra $D_{2}$. However, it will be convenient for us to have an explicit multiplicative norm on $D_{2}$.

For an arbitrary dual number $a+\epsilon b$ we let $\|a+\epsilon b\|=\frac{1}{3} \max (|a|,|b|)$.

Lemma 1. The norm $\|a+\epsilon b\|=\frac{1}{3} \max (|a|,|b|)$ on $D_{2}$ is multiplicative, that is, $\|x y\| \leqslant$ $\|x\|\|y\|$ for all $x, y \in D_{2}$.

Proof. Let $x=a+\epsilon b, y=a^{\prime}+\epsilon a^{\prime}$. Then $x y=a a^{\prime}+\epsilon\left(a b^{\prime}+a^{\prime} b\right)$. But then $\|x y\|=$ $\frac{1}{3} \max \left(\left|a a^{\prime}\right|,\left|a b^{\prime}+a^{\prime} b\right|\right)$. The numbers $|a|,|b|$ do not exceed $\max (|a|,|b|)$ and the same is true for $a^{\prime}, b^{\prime}$. This is why $\frac{1}{3} \max \left(\left|a a^{\prime}\right|,\left|a b^{\prime}+a^{\prime} b\right| \leqslant \frac{1}{3} \cdot 3\|x\|\|y\|\right.$ and hence, $\|x y\| \leqslant\|x\|\|y\|$.

By means of this norm we can construct the "standard" differential calculus for the functions over $D_{2}$. The notions of the limit (in the norm sense), of the derivative, indefinite and definite integrals (the latter is introduced as the limit of integral sums) are introduced. All usual properties are preserved. Then one can introduce functional series, power series, etc. Here we do not develop this direction, but we shall employ them in another place.

We provided a standard definition of a manifold over the algebra $D_{2}$. The corresponding $D_{2}$-structure will be called non-degenerate. But it turns out that it it natural to consider a more general case. For instance, this is needed while considering factor spaces of Lie groups and isotropic spaces. At that one can employ the notion of $\epsilon$-structure on a manifold, which is the field of linear operators in tangents spaces on the manifold, whose square vanishes (some geometricians prefers to speak about a field of affinors). In this way one introduces the field $P$ of the images of these operator as well as the field of their kernels and we obtain two distributions on the manifold. At that the issue on the integrability of these distributions arises but we do not dwell on it in details.

The presence of $D_{2}$-structure on a manifold $M$ is equivalent to the existence of an atlas on $M$ such that in its charts the above introduced linear operator $\mathcal{E}$ in the tangent spaces has constant matrices. The images of these linear operators $\mathcal{E}$ give a distribution on $D_{2}$-manifold of a half of the dimension in the non-degenerate case, which is integrable by the definition of $D_{2}$-manifold and in this way it defines a bundle usually called canonical. At that, all $D_{2^{-}}$ diffeomorphisms, that is, one-to-one and mutually $D_{2}$-smooth mappings, preserves this bundle. It is an important invariant in the category of $D_{2}$-manifolds. 
As a $D_{2}$-smooth curve on a manifold $M$, we mean a $D_{2}$-smooth mapping $\gamma: U \rightarrow M$, where $U \subset D_{2}$ is some open subset in $D_{2}$. As $U$, one can choose some ball in the sense of the above introduced norm.

The notion of a tangent vector is introduced in the natural way. For instance, we can define it as a differentiation of the germs of $D_{2}$-smooth functions at a given point or via the equivalence relation of $D_{2}$-curve at this point. We obtain the tangent space $T_{x}(M)$, which naturally has the structure of a free $D_{2}$-module.

In the natural way we define then $D_{2}$-differential forms and other objects in the standard set of the differential geometry on a $D_{2}$-manifold. We recall that at that, the multiplication by $\epsilon \in D_{2}$ in the tangent space is considered as the linear operator $\mathcal{E}$.

Then, in the natural way we introduce the tangent bundle of an arbitrary $D_{2}$-manifold $M$ and this bundle can be also regarded the tangent bundle of the manifold $M$ if we ignore its $D_{2}$-structure. Then a $D_{2}$-vector field over $M$ is the $D_{2}$-smooth section of this bundle. The set of all $D_{2}$-vector fields on the manifold form the Lie algebra over $D_{2}$. Arbitrary $D_{2}$-vector fields on the manifold $M$ can be considered as infinitesimal transformations of this manifold preserving its $D_{2}$-structure.

\section{ON DIFFERENTIAL EQUATIONS WITH DUAL INDEPENDENT VARIABLE}

Here we consider the issue on the Cauchy problem in $D_{2}$-case.

Theorem 1. Let a function $f(\tilde{x}, \tilde{u})$ be $D_{2}$-smooth in some neighbourhood of a point $\left(\tilde{x}_{0}, \tilde{u}^{0}\right)$. Then the Cauchy problem

$$
\begin{aligned}
& \frac{d \tilde{u}}{d \tilde{x}}=f(\tilde{x}, \tilde{u}), \\
& \left.\tilde{u}\right|_{\tilde{x}=\tilde{x}_{0}}=\tilde{u}^{0},
\end{aligned}
$$

is uniquely solvable in some neighbourhood of the point $\tilde{x}_{0}$.

Proof. The Cauchy problem can be rewritten as

$$
\tilde{u}(\tilde{x})=\tilde{u}_{0}+\int_{\tilde{x}_{0}}^{\tilde{x}} f(\tilde{x}, \tilde{u}) d x .
$$

This relation is considered in $D_{2}$-domain and the notion of the integral in this domain was discussed above. Then we can follow, for instance, the scheme of the proof in book by V.I. Arnold [8], which is based on the Picar mapping and on applying the iteration process or, the same, the fixed point theorem. All arguments are immediately extended to our situation if we employ the above introduced multiplicative norm for the dual numbers instead of the absolute value. There is no need to reproduce here all arguments from [8].

We can continue studying the differential equations with the dual independent variable following, for instance, the lines of book [8] for the real independent variable. Here are some results of such kind. We do not provide the proofs since they literally reproduce the proof in [8] up to the replacement of the absolute value of a real number by the norm of a dual number.

Theorem 2. (On dependence on a parameter) For each $D_{2}$-smooth function $f(x, u, \alpha), a$ Cauchy problem continuously depending on a parameter $\alpha$ (not necessarily small)

$$
\begin{aligned}
& \frac{d \tilde{u}}{d \tilde{x}}=f(\tilde{x}, \tilde{u}, \alpha), \\
& \left.\tilde{u}\right|_{\tilde{x}=x_{0}}=\tilde{u}_{0}(\alpha)
\end{aligned}
$$

is uniquely solvable in some neighbourhood of the point $\tilde{x}_{0}$ and the solution is continuous in the parameter $\alpha$. 
If the data of the Cauchy problem depend smoothly on the parameter $\alpha$, then the solution to this problem also depends smoothly on this parameter.

Of course, the theorem on continuous or smooth dependence of the Cauchy problem on the initial data is true; the initial data can be regarded as the parameters.

The theorem on the maximal continuation of the solutions is also true (up to the boundary of the domain, in which the equation is defined). The proof is based on Theorem 1, by which the solution exists in a neighbourhood of each point in the admissible domain. Then we take the union of all such neighbourhoods and choose its connected component containing the point given by the initial condition in the Cauchy problem. The solution in this domain is obviously maximally continued.

It is of course true that for a linear differential equation the solutions are defined there, where the right hand sides of the equation are defined. In other words, almost all statement of the classical theory of ordinary differential equations are extended almost immediately to the case of approximate equations and their approximate solutions. In the same way we immediately obtain the criterion of approximate invariance of the equations formulated above.

\section{ON $D_{2}$-LIE THEORY}

Our next step is to introduce the notion of $D_{2}$-Lie group. A Lie group $G$ over $D_{2}$ are Lie groups having $D_{2}$-structure on the Lie group $G$ as on the manifold and on the mappings involved in the standard definition of Lie group (that is, the mapping of group multiplication and the mapping of the inverse element are to be $D_{2}$-smooth). The tangent space of $D_{2}$-Lie group at its unity naturally has the structure of $D_{2}$-Lie algebra, that is, of Lie algebra being $D_{2}$-module as it was said above. Finally, in the standard natural way we define the notion of $D_{2}$-action of $D_{2}$-Lie group on $D_{2}$-manifold. This action is defined by a $D_{2}$-smooth mapping $G \times M \rightarrow M$ under standard relations defining the action of the group on the set. In particular, we have a standard definition of a one-parametric group in the category of $D_{2}$-objects; the parameter of the subgroup can take the values in $D_{2}$ although sometimes one can restrict himself by the real values of the parameter.

If on a $D_{2}$-manifold, a $D_{2}$-smooth one parametric transformations group is defined, then the tangent vectors of its orbits give rise to a $D_{2}$-vector field (speed field). Let us prove that the opposite is also true: to each vector field on a $D_{2}$-manifold $M$ a local one-parametric over $D_{2}$ and two-dimensional over $\mathbb{R}$ transformations group of this manifold $M$ is associated. This gives us an analogue of the classical theorem by S. Lie.

Proposition 1. Let $\mathcal{X}(z)$ be a smooth $D_{2}$-vector field defined in some open subset $U \subset D_{2}^{n}$. Then there exists a local one-parametric transformations group of some open subset $W \subset D_{2}^{n}$, whose tangent vector field coincides with $\mathcal{X}$.

If $\mathcal{X}$ is a vector field on a compact $D_{2}$-manifold $M$, to this field, a one-parametric transformations group of the manifold $M$ is associated.

The proof follows the standard process of integrating the corresponding differential equation; we note that the parameter in this equation ranges in the algebra $D_{2}$ or in some open subset in it. In the local case the base is unique solvability theorem for the Cauchy problem, see Section 5. Concerning the global case, in view of the considered local case, standard arguments used in the classical Lie theory are applicable.

By standard arguments one can show that as in the classical case, in the category of $D_{2}$-Lie groups and $D_{2}$-Lie algebras there is a correspondence between (local) $D_{2}$-Lie subalgebras and $D_{2}$-Lie subgroups. In the present paper we do not try to describe all options arising while considering the category of $D_{2}$-Lie groups and related objects. This is why we restrict ourselves by counting only some important steps in studying these rather new objects. 
It is natural to consider closed $D_{2}$-Lie subgroups $H \subset G$ and the corresponding homogeneous spaces. But the factor-space $G / H$ not always possesses a natural $D_{2}$-structure. More precisely, it possesses some $\epsilon$-structure brought by the exponential mapping and induced by the natural $D_{2}$-structure on the fact space of the corresponding Lie algebras induced by the operator $\mathcal{E}$. At that we get not necessarily non-degenerate $D_{2}$-structure and this is why we have consider also degenerate $D_{2}$-structures on the manifolds. For instance, let $H$ be a connected Lie subgroup of some $D_{2}$-Lie group corresponding to the abelian Lie group $\operatorname{Im} \mathcal{E}$ (this algebra is discussed in more details in anothr paper by the author) and assume that this subgroup is closed; for instance, this is the case if $G$ is a connected nilpotent Lie group. Then on the factor-space $G / H$ the trivial $D_{2}$-structure is induced; for this structure the operator $\mathcal{E}$ is zero. At that, the $D_{2}$-structure on the Lie subgroup $H$ is also trivial. But if on some closed Lie subgroup $F$ a non-degenerate $D_{2}$-structure is induced (that turns out not very often), then on the factor space $G / F$ we get a full-fledged non-degenerate $D_{2}$-structure.

\section{On APPROXIMATE DIFFERENTIAL EQUATIONS}

Following the above mentioned principle by A. Grothendieck, the above given definitions allow us to reformulate the method for analyzing differential equations with a parameter in terms of approximation and $o(\epsilon)$ in a new, more natural geometric form. Moreover, here we obtain the justification of the correspondence principle formulated in the beginning of the paper. Let us specify this method.

Assume that we are given some differential equation depending on the parameter

$$
\frac{d u}{d x}=f(x, u, \epsilon)
$$

and some initial condition $\left.u\right|_{x=x_{0}}=u^{0}(\epsilon)$ In fact, even the independent variable could be assumed to be depending on the same parameter $\epsilon$ (see below). We shall not dwell on other possible generalizations.

We consider the function $f$ and the solution $u$ up to $o(\epsilon)$. In [1] and consequent works there was described the method for calculating approximated solutions and symmetries groups of the corresponding differential equations.

Let us consider this method for the above mentioned equation. We let $u(x, \epsilon) \approx u_{0}(x)+\epsilon u_{1}(x)$ to be the unknown approximate function. We have

$$
f(x, u, \epsilon) \approx f_{0}\left(x, u_{0}(x)+\epsilon u_{1}(x)\right)+\epsilon f_{1}\left(x, u_{0}(x)+\epsilon u_{1}(x)\right) .
$$

Rewriting this equation up to $o(\epsilon)$, we obtain the approximate differential equation:

$$
u_{0}^{\prime}(x)+\epsilon u_{1}^{\prime}(x)=f_{0}\left(x, u_{0}(x)\right)+\epsilon\left(f_{0}\right)_{u}^{\prime}\left(x, u_{0}(x)\right) u_{1}(x)+\epsilon f_{1}\left(x, u_{0}(x)\right)+0 .
$$

Equating like terms (w.r.t. $\epsilon$ ), we obtain the system of equations:

$$
\begin{aligned}
& u_{0}^{\prime}=f_{0}\left(x, u_{0}(x)\right), \\
& \left.u_{1}^{\prime}(x)=\left(f_{0}\right)_{u}^{\prime}\left(x, u_{0}(x)\right)\right) u_{1}(x)+f_{1}\left(x, u_{0}(x)\right) .
\end{aligned}
$$

The first equation is the initial exact equations corresponding to $\epsilon=0$.

The second equation is found under the assumption that the function $u_{0}$ is found by the first equation. As a result, we obtain the approximate solution

$$
u(x) \approx u_{0}(x)+\epsilon u_{1}(x) .
$$

The initial condition in the Cauchy problem can be taken into consideration in a standard way and we do not dwell on this. 
Then we find the symmetries of the considered differential equation with the parameter $\epsilon$. In order to do it, we employ "approximate" vector fields

$$
\mathcal{X}(x, \epsilon) \approx \mathcal{X}_{0}(x)+\epsilon \mathcal{X}_{1}(x) .
$$

The approximate invariance condition for the differential equation is of the form

$$
\mathcal{X}(x, \epsilon) \cdot f(x, u(x)) \approx 0 .
$$

Applying the same method as above in solving the differential equation, we can obtain the method for successive finding the vector field $\mathcal{X}_{0}$ and $\mathcal{X}_{1}$ (more precisely, their components). Thus, we described briefly the method proposed first in [1].

Now we consider the modification of this method employing the above described geometric constructions. We shall obtain a more brief conceptual approach.

From the equation $u^{\prime}=f(x, u, \epsilon)$ we pass to its dual continuation:

$$
\tilde{u}^{\prime}=\tilde{f}(\tilde{x}, \tilde{u}) .
$$

We write the initial condition as

$$
\left.\tilde{u}\right|_{x=x_{0}}=\tilde{u}^{0}=u_{0}^{0}+\epsilon u_{1}^{0} .
$$

The objects with tilde can be regarded as exact on the manifold over the algebra $D_{2}$. Here the "approximate" differential equation is written as exact. The same concerns the Cauchy problem. This is why, applying the classical results on the differential equation to the manifold over $D_{2}$, we arrive at a conceptual proof for main general results of the theory of approximate solutions to differential equations. In particular, the following theorem holds.

Theorem 3. (on unique solvability of Cauchy problem) For each smooth function $f(x, u, \epsilon)$ and a smooth function $u_{0}(\epsilon)$, the Cauchy problem

$$
\begin{aligned}
& u^{\prime}=f(x, u, \epsilon), \\
& \left.u\right|_{x=x_{0}}=u_{0}(\epsilon)
\end{aligned}
$$

always has the unique solution in some neighbourhood of the point $x_{0}$.

We recall that the approximate solution is a function being a solution up to $o(\epsilon)$ of the initial differential equation with the small parameter.

In the same way we obtain other statements on approximate solutions of differential equations. In [1] and similar papers the corresponding statements followed by the proofs copying the classical ones but nevertheless needed. In our approach, often there is no need to make such proofs. The results are immediately impled by the standard non-approximate statements although applied to different in comparison with initial manifolds and to other $D_{2}$-objects.

Up to now we were in the framework of the developed in detail theory of approximate solutions and symmetries. We gave only their geometric interpretation. Now we go beyond this framework and move to much less developed domain. While studying differential equations, we propose to pass to dual (in our terminology) values not only for the equation and the unknown function but also for the independent variable. In other words, we pass completely to the category of the objects over dual numbers.

We observe that the idea to vary the independent variable was used before but in another situation. For instance, in [9] the generalization of the method in [1] is given, in which both dependent and independent variables are expanded in the parameter. We consider one more generalization of the method in [1].

We shall describe a new method based on the above introduced geometric constructions.

Here we linearize not only the equation (while finding its approximate solutions) and the differential operator, which is the infinitesimal operator of the symmetry of the equation, but 
also all other quantities involved in the equation, namely, the dependent and independent variables. At that additional functional parameters arise, which can be used for simplifying the process of calculations (vanishing by their means some terms).

We consider this generalized method at a simple example. Assume that we are given the Cauchy problem for a first order ordinary differential equation depending on a small parameter $\epsilon$ :

$$
\begin{aligned}
& \frac{d u}{d x}=f(x, u, \epsilon), \\
& \left.u\right|_{x=x_{0}}=u^{0}(\epsilon) .
\end{aligned}
$$

The considerations are made up to $o(\epsilon)$.

Employing $\epsilon$-continuation of smooth functions mentioned above, the initial differential equation is rewritten as an equation for a $D_{2}$-function of a $D_{2}$-variable with a $D_{2}$-analytic right hand side:

$$
\begin{aligned}
& \frac{d \tilde{u}}{d \tilde{x}}=f(\tilde{x}, \tilde{u}), \\
& \left.\tilde{u}\right|_{\tilde{x}=\tilde{x}_{0}}=\tilde{u}^{0} .
\end{aligned}
$$

Applying known methods of the theory of differential equations developed for real and complex domains to this $D_{2}$-equation, we shall obtain new results concerning approximate solutions. However, we should note that we in fact introduce two independent variables $x, y(\tilde{x}=x+\epsilon y)$ and we need to relate them (see below for the details), for instance, we observe that in the expression for $\frac{d \tilde{u}}{d \tilde{x}}$ the term $\frac{d y}{d x}$ ) is involved. The choice of such relation is an independent step of the described method.

Let us show how one can calculate the derivative $\frac{d u}{d z}$ of the function of dual argument $z=x+\epsilon y$. Here we shall need the formula for division of dual numbers. We note that such division is not always possible.

We have

$$
\frac{1}{a+\epsilon b}=\frac{1}{a}\left(1-\epsilon \frac{b}{a}\right),
$$

which can be checked by straightforward calculations. As $a \neq 0$, such division is possible and unique. We consider the derivative

$$
\frac{d u}{d z}=\frac{d u}{d x+\epsilon d y}
$$

Employing the aforementioned formula for the inverse number, we obtain

$$
\frac{d u}{d z}=\frac{d u}{d x}\left(1-\epsilon \frac{d y}{d x}\right)
$$

If we define a function $y=p(x)$ as a function of $x$, we obtain the formula $u^{\prime}=u^{\prime}-\epsilon p^{\prime} u^{\prime}$, where the prime stands for the derivative w.r.t. $x$. The developing and using of the mentioned new method is due to the specialists in this field.

In conclusion we observe that the passage to the dual numbers while solving and studying equations with a small parameter simplifies essentially routine calculations. For instance, assume that we need to solve the Cauchy problem for a linear second order ordinary differential equation:

$$
\begin{aligned}
& u^{\prime \prime}=(1+\epsilon) u-2 \epsilon, \\
& \left.u\right|_{x=0}=1-2 \epsilon,\left.u^{\prime}\right|_{x=0}=\epsilon .
\end{aligned}
$$

We shall solve it in $D_{2}$-domain. In fact, something like this can be done by using the symbol $o(\epsilon)$ but at that one has to control all the time the well-posedness of the simplifications. In 
the dual domain the well-posedness is attained immediately. One just need to employ several simple formulae: $e^{a+\epsilon b}=e^{a}(1+\epsilon b), \sin (a+\epsilon b)=\sin (a)+\epsilon b \cdot \cos (a),(1+\epsilon)^{d}=1+\epsilon d$, etc. In particular, solving the above problem, for the characteristic roots of the corresponding homogeneous equation we obtain the expressions $\lambda_{1,2}= \pm(1+\epsilon / 2)$. Then, employing the above formula for the exponent, for the general solution to the homogeneous equation we obtain:

$$
U_{0}=C_{1} e^{x}+c_{2} e^{-x}+\frac{\epsilon}{2}\left(C_{1} e^{x}-C_{2} e^{-x}\right) .
$$

Finding a particular solution for our simple right hand side, we find the particular solution $u^{*}=2 \epsilon$ and then the general solution to our differential equation. All calculations are pure arithmetical. Finally, two initial conditions give "exact" in dual domain but approximate in the substantive interpretation expression for the solution of the posed Cauchy problem $\left(C_{1}=\right.$ $\left.\frac{1-\epsilon}{2}, C_{2}=\frac{1-3 \epsilon}{2}\right)$. By the same scheme one can consider many other examples, including the ones considered in numerous papers devoted to solving approximate differential equations. By similar calculations many problems on calculating the symmetries for the differential equations are solved At that, considering the case $\epsilon=0$ and $\epsilon \rightarrow 0$, we select the symmetries destroyed for non-zero values of the parameter $\epsilon$. Here we do not dwell on details since the aim of the present paper was not to make such calculations but to develop a new geometric approach to such calculations.

\section{BIBLIOGRAPHY}

1. V.A. Baikov, R.K. Gazizov, N.Kh. Ibragimov. Approximate symmetries // Matem. Sborn. 136:4, 436-450 (1988). [Math. USSR-Sb. 64:2 427-441 (1989).]

2. V.A. Baikov, R.K. Gazizov, N.Kh. Ibragimov. Perturbation methods in group analysis // Itogi Nauki i Tekhniki. Ser. Sovrem. Probl. Mat. Nov. Dostizh. 34, 85-147 (1989). [J. Soviet Math. 55:1, 1450-1490 (1991).]

3. N.H. Ibragimov. CRC handbook of Lie group analysis of differential equations. V.3: New trends in theoretical development and computational methods. Taylor \& Francis Inc, Bosa Roca (1995).

4. V.A. Băkov, R.K. Gazizov, N.H. Ibragimov. Approximate groups of transformations // Differ. Uravn. 29:10, 1712-1732 (1993). [Differ. Equats. 29:10, 1487-1504 (1993).]

5. V.F. Dimenberg. Screw calculus. Nauka, Moscow (1965). (in Russian).

6. M. Pakdemirli, M. Yurusoi, I.T. Dolarci. Comparison of approximate symmetry methods for differential equations // Acta Appl. Math. 80:3, 243-271 (2004).

7. V.V. Vishnevskii, A.P. Shirokov, V.V. Shurygin. Spaces over algebras. Izd-vo KGU, Kazan (1985). (in Russian).

8. V.I. Ordinary differential equations. Regul. Chaot. Mekh., Izhevsk (2000). (in Russian).

9. Z.-Y. Zhang, X.-L. Yong, Y.-F. Chen. A new method to obtain approximate symmetry of nonlinear evolution equation from perturbations // Chinese Phys. B. 18:7, 2629-2633 (2009).

Vladimir Vital'evich Gorbatsevich,

I-626 Mir av. 116A-68, 129626, Moscow, Russia

E-mail: vgorvich@yandex.ru 